Revue des patrimoines

Le cheval et ses patrimoines (1ère partie)

\title{
De l'Académie d'équitation aux carabiniers de Monsieur. Les origines de la vocation cavalière de Saumur
}

\section{Élisabeth Verry}

\section{(2) OpenEdition}

\section{Journals}

Édition électronique

URL : http://journals.openedition.org/insitu/9965

DOI : $10.4000 /$ insitu.9965

ISSN : 1630-7305

Éditeur

Ministère de la culture

Référence électronique

Élisabeth Verry, «De l'Académie d'équitation aux carabiniers de Monsieur. Les origines de la vocation cavalière de Saumur », In Situ [En ligne], 18 | 2012, mis en ligne le 29 octobre 2012, consulté le 20 avril 2019. URL : http://journals.openedition.org/insitu/9965 ; DOI : 10.4000/insitu.9965

Ce document a été généré automatiquement le 20 avril 2019

\section{(c)}

In Situ Revues des patrimoines est mis à disposition selon les termes de la licence Creative Commons Attribution - Pas d'Utilisation Commerciale - Pas de Modification 4.0 International. 


\title{
De l'Académie d'équitation aux carabiniers de Monsieur. Les origines de la vocation cavalière de Saumur
}

\author{
Élisabeth Verry
}

1 Évoquer la vocation cavalière de Saumur semble aujourd'hui une évidence, tant l'architecture et l'image de la ville sont indissolublement liée à la présence des chevaux et de leurs cavaliers, aux institutions successives qui s'y développèrent de manière quasi ininterrompue pendant plus de quatre siècles. Leur histoire appartient autant à celle de la ville qu'à celle de l'art et des sports équestres, et bien entendu, comme en témoigne le beau musée récemment ouvert dans les murs de l'ancienne École de cavalerie, à l'histoire militaire française. Dans la même unité de préoccupations, se croisent parmi les auteurs qui ont consacré leurs travaux à ce sujet autant les plumes des officiers que celles des historiens, chacun apportant sa part à une construction historique qui a, sous l'impulsion des derniers d'entre eux, quitté peu à peu le ton de la célébration pour celui, moins fervent mais plus réaliste, de la recherche scientifique ${ }^{1}$.

2 Il est plaisant, mais bien entendu sans fondement, de faire appel aux Gaulois et aux Romains pour légitimer la vocation cavalière de Saumur ${ }^{2}$. Il est prestigieux, mais bien artificiel, d'évoquer pour servir la même cause la mémoire du roi René, même s'il est exact que se tint à Saumur, en juin 1446, à l'initiative du prince, l'une des plus belles fêtes par lui données en Anjou, connue sous le nom de «Pas du Perron », ou encore «Emprise de la Joyeuse Garde ${ }^{3}$ ». Dans la plaine de Launay, où le roi possède un château de plaisance, est alors édifié un superbe décor de bois, orné de tapisseries, devant lequel durant quarante jours excepté le vendredi, s'organisent des joutes entre " assaillants » et "tenants", ces derniers défendant un perron de marbre gardé par un nain entouré de deux lions. Des présents sont échangés, dont la nature donne idée de la magnificence des princes invités à l'événement : après les combats singuliers, les vaincus sont tenus d'offrir à leur vainqueur une pierre précieuse, ou un cheval : cinquante-quatre diamants, trentesix rubis furent, semble-t-il, alors offerts aux lauréats, tandis que les deux grands 
vainqueurs se voyaient gratifiés d'un destrier pour le premier, et d'un fermaillet de diamants pour le second.

3 Le souvenir de cette fête somptueuse demeura longtemps dans la mémoire des Saumurois. L'art équestre était alors indissociable de l'art militaire, et toujours très en honneur lors des démonstrations de prestige des souverains. Ainsi cite-t-on également en 1518 une parade accompagnée de joutes lors du passage à Saumur du roi François $\mathrm{I}^{\mathrm{er}}$. Mais rien dans ces exhibitions ne peut être retenu comme représentatif d'une spécificité locale.

4 Il en va tout autrement des deux institutions qui vont faire l'objet de cette étude, l'Académie d'équitation d'une part, dont l'histoire couvre une grande partie du XVII siècle, le régiment de Carabiniers et l'École d'équitation institués par Choiseul au XVIII ${ }^{\mathrm{e}}$ siècle, d'autre part.

\section{L'Académie d'équitation}

5 Il faut faire table rase des hypothèses qui feraient remonter à la fin $\mathrm{du}^{\mathrm{X}} \mathrm{XV}^{\mathrm{e}}$ siècle la présence d'une académie d'équitation à Saumur. En effet, cette affirmation, que l'on trouve au XIX ${ }^{e}$ siècle notamment dans les écrits du colonel Savette ${ }^{4}$, ne trouve d'appui que dans une délibération du Conseil de ville qui, le 16 août 1680, devant les graves difficultés rencontrées par l'institution, se prévaut d'en être "dans une possession continuelle depuis plus de deux cents ans $s^{5} »$. Une manière de défense qui n'a d'autre but que de fonder les droits de la ville à bénéficier du soutien royal à un moment où celle-ci est directement concurrencée par les prétentions d'Angers, dont le gouverneur, par ailleurs Grand Écuyer de France, le comte d'Armagnac, vient de confier l'Académie d'équitation à un célèbre maître de manège, François Avril de Pignerolle. Angers ne réclame alors rien moins que la fermeture de l'école de Saumur 6 , et la vive réaction des Saumurois est bien compréhensible.

6 C'est bien plutôt à la dynamique nouvelle engendrée à la fin du XVI ${ }^{e}$ siècle par la politique active du gouverneur Duplessis-Mornay qu'il faut attribuer la naissance d'un cours d'exercices équestres ${ }^{7}$, qui prendra plus tard le nom d'académie d'équitation. L'Académie protestante créé par lui dès 1593 connaît un rapide essor, faisant bientôt converger vers Saumur des étudiants issus des diverses régions du royaume et même de l'étranger. Les jeunes élèves se voient proposer, en plus de la formation religieuse et intellectuelle dispensée par le collège, des entraînements physiques ou savants alors corollaires de toute éducation soignée. En matière d'équitation, leur vie itinérante rend indispensable une bonne maîtrise des techniques d'art équestre.

7 Des témoins attestent dès le début du XVII ${ }^{\mathrm{e}}$ siècle la présence de maîtres d'équitation : en 1609-1610, le sieur de Croydebert ${ }^{8}$ évoque l'existence d'un manège et cite le nom de l'écuyer, monsieur de Jolivoix, qu'il qualifie d'«ami", sans toutefois prononcer le nom d'académie ; en 1614, le voyageur Just Zinzerling confirme, dans son Itinerarium Galliae ${ }^{9}$, que «beaucoup d'Allemands, de Flamands et d'Anglais fréquentent la ville à cause de l'agrément de l'endroit et d'un coût de la vie raisonnable : en conséquence, il se trouve ici des maitres dans la plupart des exercices que les étrangers ont l'habitude de pratiquer "; nettement plus tard, en 1644, la chose est encore confirmée par le savant Élie Brackenhoffer ${ }^{10}$ qui énumère « les bons maîtres d'exercice qui s'y trouvent: deux maîtres d'équitation, deux maitres de danse, deux maîtres de langue et un maître d'armes, et un 
mathématicien d'une certaine réputation ». Tout est donc en place pour que la meilleure éducation soit dispensée aux jeunes gens, nobles et bourgeois, qui fréquentent l'Académie protestante: savoir monter et soigner les chevaux, connaître des rudiments d'art équestre ${ }^{11}$; pratiquer la danse, les armes, pour compléter leur formation mondaine et sportive. Les langues, peu enseignées à cette époque, figurent également au programme des cours complémentaires, cas particulier à Saumur où se trouvent regroupés de nombreux étrangers ${ }^{12}$; de même, un enseignement des mathématiques est proposé, supplément original car cette science était alors très peu prisée.

8 Les maîtres d'exercice, qui participent de la réputation de la ville, reçoivent son soutien mais ne sont pas à cette époque rétribués par elle, quoiqu'ils y soient bien accueillis. La municipalité met à leur disposition ses écuries, situées près de l'église Saint-Nicolas et donnant sur la "cour Couronne » qui sert de carrière. Elle leur permet aussi d'utiliser comme manège les bâtiments des anciennes halles, alors fort délabrés ${ }^{13}$. Les maîtres d'exercice ont leur siège dans un petit pavillon situé dans le Grand Jardin ${ }^{14}$ et dans lequel des leçons ont pu être aussi dispensées. Après monsieur de Jolivoix, le nom d'un autre maître de manège apparaît au milieu du XVII ${ }^{\mathrm{e}}$ siècle : il s'agit de Hallot, ou du Hallot. En 1644, lors de la venue d'Henriette de France, il reçoit l'ordre de faire monter à cheval ses " escholiers " pour rendre leurs devoirs à la reine. Les élèves ne sont alors pas plus d'une vingtaine. Mais en 1648, le maître part pour Angers où il fonde une académie d'équitation plus prospère.

9 L'activité de l'Académie d'équitation va connaître ensuite une brève embellie avec Pierre Gautier de Saint-Wal, dont la présence est attestée à Saumur à compter de 1668. J.-H. Denécheau a établi, d'après l'état-civil réformé, que ce maître exerce au moins à partir du début de l'année 1668, et qu'il est allié à Du Halot, le précédent maître, puisqu'il est l'époux de sa fille Judith ${ }^{15}$. Il porte le titre d'écuyer «de la Grande écurie du roi », en référence à l'institution royale chargée de l'enseignement de l'art équestre de laquelle il se réclame. S'il est certain qu'il possède les connaissances requises, toutefois le titre qu'il porte ne doit pas faire illusion : dès cette époque, l'office d'écuyer est une charge vénale, et les nombreux abus de son emploi conduiront Louis XIV en 1669 à en confier l'attribution exclusive au Grand Écuyer de France. Gautier de Saint-Wal exerce sa charge durant trois ans. Puis, peut-être à cause de la difficulté de faire vivre un tel office à Saumur, il part servir dans les armées de l'Électeur du Palatinat, alors allié de la France, et le procès-verbal de l'assemblée des habitants ne peut que regretter le 19 novembre 1673 les conséquences funestes de ce départ ${ }^{16}$. Mauvaise décision. La campagne tourne court et Saint-Wal cherche à récupérer son emploi. Les Saumurois sont réticents : ce premier revirement, sa qualité de protestant - la méfiance du roi à leur égard grandit et de plus en plus de charges leur sont fermées -, ses liens avec son beau-père Du Halot, maître de manège à Angers, leur font redouter un nouvel échec. C'est pourquoi ils portent leur choix sur une autre candidature, celle d'un natif de la ville, le sieur Lessigny de Maliverné, par ailleurs ancien élève de Saint-Wal. Ils font également réparer les locaux dédiés à l'Académie et lui octroient diverses aides en nature pour lui permettre d'équilibrer son activité ${ }^{17}$. C'était compter sans la résistance de Saint-Wal : le comte de Comminges lui-même, gouverneur de Saumur, impose son rétablissement en 1677. Mais ce retour est de courte durée : en 1680, Pierre Gautier de Saint-Wal est contraint de cesser son activité, dont l'exercice est interdit aux réformés. Le Conseil de ville ne manque pas d'ironiser, indiquant que «le maître de manège est obligé depuis quelques mois d'en 
cesser l'exercisse par ordre de Sa Majesté, à cause qu'il est de la RPR (dont nous avions informé monseigneur notre gouverneur) ${ }^{18}$ ".

Malgré ces difficultés, les Saumurois n'entendent pas renoncer. Par délibération du 16 août 1680 , le Corps de ville prend la décision d'engager à ses frais un nouveau maître de manège, Jacques Dupré, fils d'un avocat de la ville, qu'il aide à racheter l'équipage de Saint-Wal et à qui il accorde une rétribution de 150 livres par an et le titre d'« écuyer de la ville de Saumur ${ }^{19}{ }$. L'Académie d'équitation est donc devenue désormais une institution municipale. Néanmoins l'activité peine à survivre. Un certain Duvernet est recruté en 1681, qui s'avère incompétent. En 1697, l'Académie est fermée, et ses écuries servent à loger les dragons de passage. L'année suivante, l'assemblée générale des habitants tente une nouvelle relance: Jacques Dupré se voit gratifié de 3000 livres pour rétablir l'académie, et aménager de nouveaux locaux dans le quartier de la Maremaillette ${ }^{20}$. Comme il n'y a plus d'étrangers à Saumur, on compte sur la fréquentation d'une clientèle bourgeoise et la rétribution à verser au maître est fixée à 20 livres par mois. Un tarif élevé qui ne dut pas susciter une demande suffisante puisqu'au XVIII ${ }^{e}$ siècle, si l'on trouve encore trace de maîtres d'exercice en mathématiques, ou en écriture, il n'est en revanche plus question de maître de manège. L'Académie d'équitation de Saumur a bel et bien vécu.

\section{Le régiment des « Carabiniers de Monsieur »}

11 Il fallut attendre plus d'un demi-siècle pour que l'équitation trouve à nouveau à Saumur droit de cité, dans un développement d'une tout autre envergure. La décision de Choiseul d'y établir à compter de 1763 le régiment des Carabiniers, attribué par ordonnance du 13 mai 1758 au comte de Provence, frère du futur Louis XVI, est décisive et inscrira durablement l'équitation au centre de l'activité saumuroise.

Jean-Pierre Bois en a livré en 1980 une magistrale étude, qui ne sera reprise ici qu'à grands traits ${ }^{21}$.

13 Le régiment des Carabiniers est un corps d'élite à la réputation glorieuse ${ }^{22}$. Il vient de s'illustrer à de nombreuses reprises durant la guerre de Sept ans, et son commandant, le comte de Gisors, a été tué en 1758 à Krefeld en chargeant l'infanterie hanovrienne. Lui a succédé le marquis de Poyanne, officier valeureux, plusieurs fois blessé, qui a gagné le grade de lieutenant général des armées du roi, et commandera le régiment jusqu'à sa mort en 1781.

L'installation des Carabiniers à Saumur n'est pas le fruit du hasard. La fin de la guerre franco-prussienne conduit le ministre Choiseul à réorganiser profondément les armées. Le régiment des Carabiniers est cantonné dans la généralité de Tours, une première brigade s'installe à Saumur en 1763 venant du Mans, une seconde brigade précédemment installée à Angers la rejoint l'année suivante ${ }^{23}$.

Par ailleurs, Choiseul décide d'accompagner la réorganisation des troupes par la création de cinq écoles d'équitation réparties à travers le royaume. Il s'agit en effet de donner un socle commun de pratiques équestres aux membres des régiments de cavalerie, qui n'étaient soumis jusqu'alors à aucun enseignement spécifique ${ }^{24}$. Pour la région, le choix se porte d'abord sur La Flèche, où sont offerts les bâtiments de l'ancien collège abandonné par les Jésuites. Mais quand Choiseul vient visiter les lieux en 1764, il change d'avis. La Flèche sera transformée en école militaire préparatoire, et l'école d'équitation s'installera à Saumur, où siège désormais l'état-major des Carabiniers. 

princier : il passe sous un arc de triomphe monté dans l'île du Parc, devant le débarcadère de son bateau. Les rues dépavées sont recouvertes de sable et l'on déploie à grands frais des illuminations nocturnes. Le régiment lui est présenté au grand complet, 810 hommes et 780 chevaux s'étant ajoutés aux deux brigades déjà présentes, et à l'état-major. La ville se serre pour loger tout ce monde. Si les hommes se casent tant bien que mal, les chevaux restent pour la plupart au piquet sur le Chardonnet. Néanmoins le ministre se déclare satisfait et conforte la présence de la cavalerie à Saumur.

Déjà les premières constructions commencent à s'élever. Le vaste périmètre du Chardonnet et du Breil, qui s'étendait du Pont Fouchard à l'embouchure du Thouet, offre un espace approprié, idéalement situé à proximité de la ville. Dès 1764, à partir de plans dressés par l'ingénieur Louis-Alexandre de Cessart, un premier manège est édifié au Chardonnet, sur l'emplacement de l'actuel manège des Écuyers ${ }^{25}$. J.-H. Denécheau signale à bon droit que l'aménagement du site a suscité dès cette époque des projets ambitieux, élaborés autour de 1765 par les ingénieurs de la généralité de Tours ${ }^{26}$, mais qui restent lettre morte, faute d'argent bien sûr. L'aménagement se poursuit plutôt au coup par coup. En 1766, François-Michel Lecreux, successeur de Cessart, élève entre l'actuelle rue Beaurepaire et la rue Saint-Nicolas de longues et étroites écuries couramment appelées "Écuries des Cent-Chevaux» ou "Petites-Écuries», dont il indique qu'elles ont une capacité de 120 stalles. Entre les deux bâtiments, dans l'espace aujourd'hui occupé par le hangar Bossut, prend place une carrière découverte. Enfin, de l'autre côté du Chardonnet, l'ingénieur Lecreux, reprenant des plans tracés par Cessart, achève un hangar massif que l'on appelle naturellement «Grandes écuries » (aujourd'hui écuries de la Moskova) d'une contenance de 240 stalles. Plus vaste et plus soigné que le premier édifice, ce bâtiment témoigne de l'ambition grandissante des autorités pour la place de Saumur.

Mais si les chevaux trouvent place dans ces deux édifices, la décision la plus importante est sans conteste celle de la construction de la caserne, qui intervient à compter de 1767. Le parti architectural est signe de temps nouveaux. En effet, alors que la tradition veut jusqu'alors que les quartiers destinés à la cavalerie comportent, répartis autour d'une cour centrale, un vaste et haut rez-de-chaussée destiné au logement des chevaux, tandis que les hommes sont logés à l'étage et les officiers dans un pavillon attenant, la caserne de Saumur est entièrement dédiée au logement des hommes, officiers et cavaliers, et aux besoins de l'apparat et de la gestion du régiment. L'ingénieur en chef de la généralité, Jean-Baptiste de Voglie, supervise les travaux, conduits sur place par l'ingénieur Lecreux secondé par Jean-François Lamandé. Le chantier, attribué à l'entreprise Cailleau, est rondement mené ; en pierre de Champigny, tuffeau et ardoise, les travaux sont achevés dans le courant de l'année 1770. Malgré la qualité de sa construction, immédiatement apparait la plus grande faiblesse du projet : trop peu surélevé par rapport au niveau du fleuve, la caserne est gravement inondée à deux reprises, en 1770 et 1783. Son aménagement intérieur est laborieux : plusieurs marchés témoignent de l'ameublement et de la décoration progressive des lieux, qu'il s'agisse des chambres des officiers, pourvus de lits à baldaquins et de rideaux, ou des chambres où les soldats dorment par quatre, sauf si, mariés, ils ont le privilège de chambres particulières. Au total, ce sont plus de 400 personnes qui sont ainsi hébergées.

La construction de la caserne consolide incontestablement la présence de la cavalerie à Saumur. Un dernier bâtiment couvert, aujourd'hui «manège Montbrun », est construit le long de la Loire, tandis qu'un magasin à avoine est adossé à la levée, sur l'emplacement de 
l'ancienne poudrière. Peu avant la Révolution, s'ajoutent à cet ensemble deux écuries privées pour répondre aux besoins que le manque d'argent public ne permet plus de couvrir. Construites de 1786 à 1788 par l'entrepreneur Cailleau et le Saumurois JeanMartin Fournier de Boisayrault, elles sont louées aux régiments ${ }^{27}$.

Toutefois la présence des militaires est sujette à de fortes variations. Le régiment des carabiniers prend souvent la route de l'est, il est cantonné en 1772 à Metz et à Lunéville, revient pour partie à Saumur entre 1775 et 1781. Il porte désormais le nom de « régiment des Carabiniers de Monsieur ", comme l'implique la nouvelle dignité du comte de Provence, frère du roi, depuis l'avènement de Louis XVI. En 1783, c'est le régiment d'Orléans-Cavalerie qui s'installe dans les murs, les Carabiniers étant quant à eux de retour en 1784. Ils y demeurent jusqu'au printemps 1788, date à laquelle, après une ultime réorganisation qui les divise en deux régiments, ils sont renvoyés dans l'est. Le 7 avril 1788, le marquis de Chabrillant, colonel en exercice, dépose dans l'église Saint-Pierre les deux anciens étendards qui sont accrochés aujourd'hui encore à l'entrée du chœur. Saumur ne perd pas pour autant toute fonction militaire : à la veille de la Révolution, y résident trois escadrons du Royal Roussillon, $11^{\mathrm{e}}$ régiment de cavalerie, comptant 553 soldats.

21 Le cadre de cet article ne permet pas de décrire longuement les conditions de vie et d'exercice des régiments de cavalerie à Saumur en cette fin du XVIII ${ }^{e}$ siècle. On en trouvera les détails dans les travaux de J.-P. Bois et de J.-H. Denécheau, déjà cités. Toutefois, il paraît important d'observer deux points particuliers, dont les développements seront essentiels au siècle suivant: le fonctionnement de l'école d'équitation, d'une part, l'insertion des militaires dans la ville, d'autre part.

L'existence d'une école d'équitation, voulue par Choiseul, ne doit pas faire illusion. Il ne s'agit pas d'un établissement largement ouvert, ni fédérant, par une organisation rigoureuse, la formation de l'ensemble des cavaliers des armées françaises. La création d'un enseignement à Saumur ne correspond qu'aux premières tentatives de création d'un enseignement équestre spécifiquement militaire, comme l'a très bien montré J.-P. Bois ${ }^{28}$. Durant les années de son existence au XVIII siècle, l'école est au service du corps des Carabiniers, et nulle trace n'apparait, malgré les souhaits de principe, de la présence de cavaliers venus d'autres régiments. Un enseignement de tenue à cheval est donné aux hommes de troupe par les bas-officiers, suivant des formes rustiques dont témoignent les manuels de l'époque ${ }^{29}$. Un cours d'hippiatrie est dispensé, probablement à l'infirmerie vétérinaire située dans l'île du Parc. Des exercices de haute école sont pratiqués, dont témoignent la présence d'un maître de manège et de chevaux gris destinés à l'exercice. Le contenu de l'apprentissage est progressif : l'instruction débute sur des chevaux de bois, puis les cavaliers apprennent à manœuvrer par deux, puis quatre, puis à évoluer en différentes formations. Ils apprennent à charger le sabre haut, à manier le pistolet, et toutes attitudes propres à conforter leur position au combat, et à y habituer leurs chevaux. Somme toute, et d'après les témoignages recueillis par J.-H. Denécheau, l'instruction donnée, à défaut d'être brillante, semble solide et profitable ${ }^{30}$. Mais l'absence d'autonomie de l'école par rapport au régiment fait aussi sa fragilité : lorsque le régiment est absent, ou se déplace, elle disparait. Aucun exercice n'est signalé après le départ de la troupe en 1788 , le nom même d'école d'équitation n'est plus prononcé.

23 Dans la ville, le régiment s'insère progressivement, à mesure que les conditions d'accueil s'y améliorent, la période la plus marquante semblant être celle des années 1784-1788. Le régiment est très nombreux, puisque son effectif théorique est de 80 officiers et 
1560 hommes de troupe répartis en 5 brigades et 30 compagnies, chacune comprenant un capitaine, un lieutenant et 52 carabiniers. Il ne faut pas oublier que les accompagnent 1200 chevaux de combat, et quelques dizaines de chevaux d'appoint et d'exercice. Toutefois les effectifs ne sont jamais complets, en raison des congés, maladies, et désertions qui se chiffrent couramment à une centaine de manquants. Les carabiniers sont aisément identifiables à leur uniforme, établi par l'ordonnance du 21 décembre 1762 qui a consacré l'abandon de l'antique cuirasse en fer bruni: habit de drap bleu, avec revers rouges et parements en galons d'argent ; culotte et gilet blanc; tricorne noir bordé d'argent ; bottes noires. Ainsi vêtus, montés sur des chevaux noirs et hongres en principe de race Holstein, ils ont fière allure.

Bon nombre d'officiers délaissent la caserne pour s'installer en ville avec femme et enfants. Des relations mondaines s'établissent, des fêtes et réceptions s'organisent ${ }^{31}$. Quand, en 1785, est fondée une société par actions pour construire une nouvelle salle de spectacle, des officiers en souscrivent des parts. La présence militaire suscite le développement de l'hôtellerie, tant sur le plan de la location d'appartements que de l'activité des débits de boisson. Plusieurs officiers s'établissent, faisant construire des maisons et des hôtels. Un artisanat nouveau (selliers, éperonniers, armuriers) apparaît, tandis que l'artisanat traditionnel voit son activité démultipliée. La ville vit en partie de la présence militaire. Un officier le souligne, après le départ des Carabiniers en 1783 : «en renonçant à mettre des troupes [à Saumur], ce serait ruiner ce pays-là, qui s'est beaucoup augmenté en artisans depuis 1763, que nous y avons été en quartier pour la première fois 32 ».

25 Mais la présence des militaires ne présente pas que des avantages. Ils sont malades, fréquemment atteints de maladies vénériennes, si bien qu'une infirmerie spécialisée est finalement aménagée en octobre 1787 dans une maison située près des grandes écuries. Les autres malades sont installés dans deux salles de l'Hôtel-Dieu qui leur sont dédiées, ne comportent que 21 places et sont constamment remplies. Les conditions de soins et d'hygiène y sont si mauvaises qu'une infirmerie de dix lits est aménagée en 1786 dans la caserne, principalement destinée aux officiers. Pourtant, la cohabitation entre les soldats et les habitants semble dans l'ensemble satisfaisante. Peu d'affaires sont portées devant les tribunaux, qui se résument à quelques larcins et bagarres, sauf les cas de désertion, sévèrement punis ; stricte séparation du militaire et du civil, crainte de la justice, ou voisinage apaisé entre les deux communautés: les relations entre militaires et civils semblent avoir à Saumur, en cette fin de XVIII siècle, trouvé un juste équilibre.

Le départ des Carabiniers en 1788 précède de peu la grande rupture de la Révolution. Le régiment lègue à Saumur un exceptionnel ensemble de terrains et d'installations dont le comte de Provence, devenu en 1814 le roi Louis XVIII, se souviendra lorsqu'il sera question de restaurer un enseignement commun à tous les corps de cavalerie qu'avait une première fois tenté Bonaparte, entre 1809 et 1814, au château de Saint-Germain en Laye. Soutenu par le duc d'Angoulême, neveu du roi et favorable à Saumur, le projet est approuvé dès la fin de l'année 1814. L'« école d'instruction des troupes à cheval » investit la ville l'année suivante, écrivant une nouvelle page de l'histoire cavalière de Saumur. Depuis cette date, malgré les aléas politiques et la formidable évolution des organisations et des techniques militaires, la Cavalerie est toujours présente à Saumur. La greffe des Carabiniers a été solide, et la vocation cavalière de la ville peut sans conteste s'enorgueillir de ses quatre siècles d'existence. 


\section{NOTES}

1. - L'histoire de l'École de cavalerie donne lieu dès la fin du XIX ${ }^{\mathrm{e}}$ siècle à de nombreuses études à valeur plus documentaire qu'historique : ce sont celles du Capitaine PICARD, L. Les origines de l'École de cavalerie et de ses traditions équestres. Saumur : S. Milon fils, 1889; du général de FORNEL DE LA LAURENCIE. L'École de Saumur. Angers : Éditions de l'Ouest, 1935 ; du colonel de cOSSEBRISSAC. «L'École de Saumur ». Revue historique de l'Armée, 1954, n³-4, p. 104-106; du général DUROSOY. Saumur. Historique de l'École d'application de l'arme blindée et de la cavalerie. Paris, 1964, rééd. 1978 ; l'histoire de l'Académie d'équitation est abordée par le colonel SAVETTE. L'Académie royale d'équitation de Saumur avant 1789. Saumur: Girouard et Richou, 1934. Dans une période récente, citons comme essentiels les travaux de: BOIS, J-P. «Le régiment des carabiniers de Monsieur à la fin de l'Ancien régime ». Revue historique des Armées, 1980, n 1, p. 29-60 ; "L'École de cavalerie de Saumur ». Archives d'Anjou, 1998, p. 117-130; la thèse d'École des chartes, encore inédite, de CONRAUX, Aurélien. L'école de cavalerie de Saumur (1814-1914). La création de l'équitation militaire, 2004 ; enfin les chapitres consacrés tant à l'Académie d'équitation qu'à l'École de cavalerie par J-H DENECHEAU sur son site Internet http://pagesperso-orange.fr/saumur-jadis/ dont il est à souhaiter qu'elles soient un jour pérennisées par une publication en forme classique. 2. - « À la belle saison, les tribus celtiques qui peuplaient la contrée, attirées par les riches herbages de la triple vallée, descendaient du plateau et accrochaient leurs tentes en peau d'auroch au flanc des coteaux rocheux... Quand les pluies d'automne, grossissant les eaux, faisaient se mêler les rivières, les tribus remontaient sur le plateau et abandonnaient la pêche pour chasser le sanglier ou les bêtes fauves dans les épaisses forêts dont les vestiges nous rappellent aujourd'hui l'étendue.... C'est dans ces exercices que les premiers cavaliers acquirent cette hardiesse équestre qui les fera plus tard tant apprécier des recruteurs romains » (Général de FORNEL DE LA LAURENCIE. L'École de Saumur. Angers : Éditions de l'Ouest, 1935, p. 9).

3. - Voir de MERINDOL, C. Les fêtes de chevalerie à la cour du Roi René - Emblématique, art et histoire. Le Léopard d'or, 1993 ; FAVIER, J. Le Roi René. Paris : Fayard, 2008 ; ROBIN, F. La cour d'Anjou-Provence. La vie artistique sous le règne de René. Paris : Picard, 1985.

4. - Colonel SAVETTE. L'Académie royale d'équitation de Saumur avant 1789. Saumur: Girouard et Richou, 1934

5. - Archives municipales de Saumur, BB2, fol.117-118.

6. - Archives municipales d'Angers, BB 95, fol. 147.

7. - Aucun lien cependant ne semble exister entre les exercices équestres pratiqués à Saumur et l'Académie protestante, et Duplessis-Mornay n'y fait aucune allusion dans son abondante correspondance. Voir DAUSSY, H., FERRER, V. (éd.). «Servir Dieu, le roi et l'État: Philippe Duplessis-Mornay, 1549-1623». Actes du colloque de Saumur, 15-13 mai 2004. Niort: Cahiers d'Aubigné, 2006 ; POTON, D. Duplessis-Mornay, 1546-1623 : le pape des huguenots. Paris : Perrin, 2006.

8. - Le sieur de Croydebert réside à Saumur dix-huit mois en 1609-1610 comme gouverneur de trois gentilshommes saintongeais. Il donne de son séjour une amusante et précieuse relation. CROYDEBERT. L'adieu facétieux du sieur de Croydebert sur son départ du lieu et ville de Saumur. Saumur : Pierre Godeau, 1611.

9. - Le Thuringien Just Zingerling séjourne à Saumur en 1614, et publie à Lyon en 1616 un guide de voyage fort précis. ZINGERLING, Just. JodociSinceriltinerariumGallae...Lugduni, apudJacobum du Creux, alias Molliard, 1616.

10. - BRAKENHOFFER, E. Voyage en France, 1643-1644, trad. Paris-Nancy, 1925, p. 207-216. 
11. - Depuis l'époque d'Henri IV, les membres de la garde bourgeoise de Saumur ont constitué la " compagnie des chevaliers de l'Arquebuse », qui défile et exécute lors des fêtes quelques figures de parade.

12. - J.-H. Dénécheau émet l'hypothèse que l'une des langues enseignées soit le français, à destination des étrangers, et l'autre l'italien.

13. - À l'emplacement actuel du hangar Bossut et du manège des Écuyers.

14. - Aujourd'hui place de l'Europe, voir J-H DENECHEAU sur son site Internet http://pagespersoorange.fr/saumur-jadis/.

15. - Pierre Gaultier de Saint-Wal (orthographié aussi de Saint-UUal ou Vual) est régulièrement cité dans le registre de l'état-civil de la communauté réformée de Saumur, dans laquelle il fait figure de notable (Archives départementales de Maine-et-Loire, I 7) : le 18 janvier 1668, il est parrain de Marie-Anne, fille du marchand apothicaire Pierre Liger; le 20 mai 1668, il baptise sa fille Marie ; le $1^{\text {er }}$ mai 1672, son fils Pierre, etc. Voir J-H DENECHEAU sur son site Internet http:// pagesperso-orange.fr/saumur-jadis/.

16. - « La ville se désertant de plus en plus parce que les estrangers n'y viennent plus faire leur exercice, comme ils avoient accoustumé, fault y avoir académie et escuier qui enseigne à monter à cheval » (AM de Saumur, BB1, fol. 103-104).

17. - Vingt charretées de foin et 1500 boisseaux d'avoine, 150 livres pour son logement, et l'exemption de tous les impôts (AM de Saumur, BB2, fol. 32 et 35).

18. - AM de Saumur, BB2, fol. 117-118.

19. - AM de Saumur, BB2, fol. 117-118.

20. - AM de Saumur, BB3, fol. 60.

21. - BOIS, J.-P. «Le régiment des Carabiniers de Monsieur à la fin de l'Ancien Régime ». Revue historique des Armées, 1980, t. 1, p. 29-60.

22. - Les carabiniers, corps spécifique monté qui précède la cavalerie au combat, à l'instar des grenadiers pour l'infanterie, apparaissent pour la première fois au XVI ${ }^{\mathrm{e}}$ siècle, puis à nouveau de manière éphémère sous Louis XIII en 1635, puis enfin sous Louis XIV qui installe une compagnie de carabiniers à la tête de chaque régiment. En 1693, ces compagnies sont réunies en une seule, le Royal Carabinier, donné au duc du Maine. Le régiment comprend, en 1758, 40 compagnies de 25 à 30 hommes chacune, réduites à 30 en 1763. Elles sont regroupées en 5 brigades.

23. - Déjà Saumur avait, durant l'été 1758 , accueilli le cantonnement du régiment de BerryCavalerie. Le registre de la paroisse Saint-Pierre (AM de Saumur, GG 28, f. 26), mentionne que le 8 août 1758 son commandant, le comte de Valbelle, a fait bénir les étendards dans l'église, chanter un Te Deum et fait tirer un feu d'artifice sur le Chardonnet.

24. - Comme l'explique J.-P. Bois, la création d'une école à Saumur correspond aux premières tentatives dans cette direction (BOIS, J.-P. «Saumur, aux origines de la cavalerie en Anjou». Archives d'Anjou, $\mathrm{n}^{\circ}$ 12, 2008, p. 121-130).

25. - Une description et des références très précises sont données sur ces sujets par Joseph-Henri Dénécheau, dans les chapitres de son étude en ligne consacrée à Saumur (http://pagespersoorange.fr/saumur-jadis/).

26. - Archives départementales de Maine-et-Loire, C 88.

27. - Voir J-H DENECHEAU sur son site Internet http://pagesperso-orange.fr/saumur-jadis/.

28. - BOIS, J.-P. « Saumur, aux origines de la cavalerie en Anjou ». Archives d'Anjou, n 12, 2008.

29. - Archives départementales de Maine-et-Loire, C 86 ; Instruction qui règle les principes nécessaires pour organiser les régiments de cavalerie à l'école d'équitation de Saumur. Saumur : Degouy, 1764.

30. - Voir notamment la relation donnée par Jean-François Bodin de la visite rendue à Saumur par l'empereur Joseph II en 1777 : «toutes les manœuvres furent exécutées avec autant de célérité que de précision; le comte [de Rochambeau, chef d'état-major du ministre de la Guerre] en témoigna sa satisfaction au commandant le marquis de Poyanne, avec les éloges les plus 
flatteurs. Ensuite, il exprima le désir de voir le régiment à pied ; on le fit aussitôt défiler devant lui, et tandis qu'il s'entretenait avec le commandant, les carabiniers se rendirent au grand trot à la caserne, changèrent d'uniforme, et lorsque le comte arriva, tout le régiment, en habit de manège, était rangé en bataille sur l'esplanade ». BODIN, J.-F. Recherches historiques sur la ville de Saumur, ses monuments et ceux de son arrondissement. Saumur : Degouy Ainé, t. II, 1814, p. 376 -384 31. - BODIN, J.-F. Recherches historiques sur la ville de Saumur, ses monuments et ceux de son arrondissement. Saumur : Degouy Ainé, t. II, 1814, p. 384-387.

32. - Lettre au général-comte de Chabrillant, mars 1783, cité par J-H. DENECHEAU sur son site Internet http://pagesperso-orange.fr/saumur-jadis/.

\section{AUTEUR}

\section{ÉLISABETH VERRY}

Directrice des Archives départementales de Maine-et-Loire e.verry@cg49.fr 